\title{
All-sky Monitoring of Variable Sources with Fermi GBM
}

\author{
Colleen A. Wilson-Hodge* \\ NASA/MSFC \\ E-mail: colleen.wilson@nasa.gov
}

Michael L. Cherry ${ }^{1}$, Gary L. Case ${ }^{1}$, Ascension Camero-Arranz ${ }^{2}$, Vandiver Chaplin ${ }^{3}$, Valerie Connaughton ${ }^{3}$, Mark H. Finger ${ }^{2}$, Peter Jenke ${ }^{4}$, James C. Rodi ${ }^{1}$, Wayne H. Baumgartner $^{5}$, Elif Beklen ${ }^{6}$, P. Narayana Bhat ${ }^{4}$, Michael S. Briggs ${ }^{4}$, Neil Gehrels ${ }^{7}$, Jochen Greiner ${ }^{8}$, Keith Jahoda ${ }^{7}$, R. Marc Kippen ${ }^{9}$, Chryssa Kouveliotou ${ }^{4}$, Hans A. Krimm $^{5}$ Erik Kuulkers ${ }^{11}$, Niels Lund ${ }^{12}$, Charles A. Meegan ${ }^{2}$, Lorenzo Natalucci ${ }^{13}$, William S. Paciesas ${ }^{3}$, Robert Preece ${ }^{3}$, Nikolai Shaposhnikov ${ }^{10}$, Gerald K. Skinner ${ }^{10}$, Doug Swartz ${ }^{2}$, Andreas von Kienlin ${ }^{8}$, Roland Diehl ${ }^{8}$, Xiao-Ling Zhang ${ }^{8}$

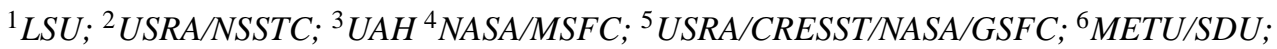

${ }^{7} \mathrm{NASA} / \mathrm{GSFC} ;{ }^{8} \mathrm{MPE} ;{ }^{9} \mathrm{LANL} ;{ }^{10} \mathrm{UMD} / \mathrm{CRESST} / \mathrm{NASA} / \mathrm{GSFC} ;{ }^{11}$ ISOC/ESA/ESAC; ${ }^{12}$ Danish

National Space Center; ${ }^{13}$ INAF-IASF

\begin{abstract}
Using the Gamma ray Burst Monitor (GBM) on Fermi, we monitor the transient hard X-ray/soft gamma ray sky. The twelve GBM NaI detectors span $8 \mathrm{keV}$ to $1 \mathrm{MeV}$, while the two BGO detectors span $150 \mathrm{keV}$ to $40 \mathrm{MeV}$. We use the Earth occultation technique to monitor a number of sources, including X-ray binaries, AGN, and solar flaring activity. Our monitoring reveals predictable and unpredictable phenomena such as transient outbursts and state changes. With GBM we also track the pulsed flux and spin frequency of accretion powered pulsars using epochfolding techniques. Highlights from the Earth Occultation and Pulsar projects will be presented including our recent surprising discovery of variations in the total flux from the Crab. Searches for quasi-periodic oscillations and X-ray bursts are also possible with GBM all-sky monitoring capabilities. With these results we show how crucial an all-sky monitor is for any future X-ray timing mission.
\end{abstract}

Fast $X$-ray timing and spectroscopy at extreme count rates

February 7-11, 2011

Champery, Switzerland

${ }^{*}$ Speaker. 


\section{Introduction}

All-sky monitors are typcially responsible for triggering observations with a main instrument, workhorse science such as monitoring source states and fluxes, and serendipitous science resulting from watching large fractions of the sky nearly all of the time. In this paper, we describe highlights of all-sky monitoring science with the Gamma ray Burst Monitor (GBM) on Fermi, including the discovery of variations in the Crab Nebula flux in hard X-rays and results from monitoring accretion-powered pulsars.

\section{Observations \& Results}

The Fermi satellite was launched on June 11, 2008. It consists of two instruments, the Large Area Telescope [1], sensitive to gamma rays from $20 \mathrm{MeV}$ to more than $300 \mathrm{GeV}$ and the Gamma Ray Burst Monitor [2], sensitive to gamma rays from $8 \mathrm{keV}$ to $40 \mathrm{MeV}$. The GBM consists of 12 $\mathrm{NaI}$ detectors (8-1000 keV) and two BGO detectors $(150 \mathrm{keV}-40 \mathrm{MeV})$. The results described in this paper are from the GBM NaI detectors.

\subsection{Discovery of decline in Crab Nebula flux using the Earth occultation technique}

The GBM instrument is not a pointed or focusing instrument. To measure fluxes from individual sources on the sky, we use the Earth occultation technique [3, 4], measuring the change in count rate as a source enters or exits Earth occultation. Our application of this technique uses a catalog of known source locations, for which Earth occultation times are calculated and a window of data containing the occultation time is extracted. These data from GBM detectors viewing the source within 60 degrees of the detector normal are fitted with a quadratic background plus a model resulting from an assumed source spectrum convolved with the changing detector response as GBM scans the sky, and the energy dependent atmospheric transmission function describing the Earth occultation. Other bright sources within the window are fit simultaneously. For the source of interest, the flux is computed by first computing the best scale factor in each energy band for all detectors in the fit, and then applying this scale factor to the reference flux, computed from the assumed model in each energy band. GBM's CTIME (0.256-s, 8 energy bands from 8-1000 keV) and CSPEC (4.096-s, 128 energy bands from 8-1000 keV) data for the NaI detectors were used in the analysis described here. The Earth occultation technique is also applicable to the the GBM BGO detectors.

As of 2011 May, GBM is monitoring a catalog of 107 sources using Earth occultation. Most of these sources were recently active X-ray binaries, plus the Crab, ten active galactic nuclei, two soft-gamma repeaters, and the Sun. In the first two years of Earth occultation monitoring with GBM, six persistent and two transient sources were detected above $100 \mathrm{keV[4].} \mathrm{More} \mathrm{than} 80$ sources have been detected below $100 \mathrm{keV}$. Preliminary monitoring results can be found on our website $^{1}$.

The most remarkable result from GBM Earth occultation monitoring was the discovery that the hard X-ray flux from the Crab nebula is not constant, and in fact, it declined by about $7 \%$

\footnotetext{
${ }^{1}$ http://gammaray.nasa.gov/gbm/science/occultation
} 
from 2008-2010 in the 15-50 keV band [5]. Figure 1 shows composite light curves combining overlapping measurements of the Crab pulsar + nebula flux from the Rossi X-ray Timing Explorer (RXTE), INTEGRAL's hard X-ray instruments ISGRI, SPI, and JEM-X, Swift, and Fermi/GBM. With this suite of instruments, we cover a broad range in hard-X/soft gamma-rays $(\sim 10 \mathrm{keV}$ to $>100 \mathrm{keV}$ ) and simultaneously address systematic uncertainties using very different types of instrumentation to demonstrate overall consistency of our Crab results. Six instruments on four satellites agree well from 2008 to 2010, with all instruments registering a 7\% decline in the 15-50 $\mathrm{keV}$ Crab nebula + pulsar flux over two years, starting at MJD 54690, and with a similar decline in the 50-100 keV band. Details of the analysis for each instrument are given in [5]. INTEGRAL/SPI data [6] are compared with the other 5 instruments for the first time in this paper. Prior to the launch of Fermi in 2008, increases and decreases in flux at a level of about 3.5\% per year were seen in the 15-50 and 50-100 keV bands, back to 1999. In more recent data, since August 2010, all of the instruments show that the Crab has stopped fading and may be beginning to brighten again.

From 1999 to 2010, the pulsed flux measured with RXTE showed only a steady decrease at $\sim 0.2 \% \mathrm{yr}^{-1}$, consistent with pulsar spin-down. The larger $\sim 3.5 \% \mathrm{yr}-1$ variations are not seen in the pulsed emission, indicating that the variations are nebular in origin [5].

In summary, the Crab is variable in hard X-rays and should not be assumed as a constant source suitable for normalizing instrument response functions or calibrating hard X-ray instruments. The variations are seen in the nebular emission, apparently resulting from changes in the shock acceleration or in the nebular magnetic field. Longer baselines and multi-wavelength observations are needed to understand the origin of these variations.

\subsection{Monitoring accreting pulsars with GBM}

As of May 2011, the GBM accreting pulsar team is currently monitoring 29 accreting pulsars and is performing daily blind searches for new accreting pulsars [10]. For both techniques, GBM data with 0.256-s time resolution for the 8-12, 12-25, and 25-50 keV bands are used. First the background data are fitted with a spline model that accounts for Earth occultation steps from bright sources. Data including solar flares, gamma ray bursts, solar panel occultations and any other problems are excluded from fitting. The remaining data result in typical exposure times of 40 ks per day, although for some sources the exposure time can reach $70 \mathrm{ks}$ per day. For the blind search, power spectra are generated for each of 24 galactic longitude bins plus separate bins for the Small and Large Magenllanic Clouds. The algorithm reports peak powers with frequencies and the galactic longitude to find new accreting pulsars and new outbursts of known pulsars that are not currently being monitored. Source specific searches are being performed for 29 sources $^{2}$, searching over a relatively narrow range of frequencies (and frequency derivative for some sources). Orbital parameters are accounted for if known. GBM has the advantage of long observing times and continuous monitoring for these sources.

A recent highlight from GBM pulsar monitoring is the detection of an unusually bright flare near apastron in the $\sim 41.5$ day orbit of GX 301-2 [11], shown in Figure 2. GX 301-2 comprises an early B-type companion and a 680-s pulsar. Typically, GX 301-2 has a bright flare near periastron and a weak flare near apastron. The bright apastron flare detected with GBM coincides with the

\footnotetext{
${ }^{2}$ http://gammaray.nasa.gov/gbm/science/pulsar
} 

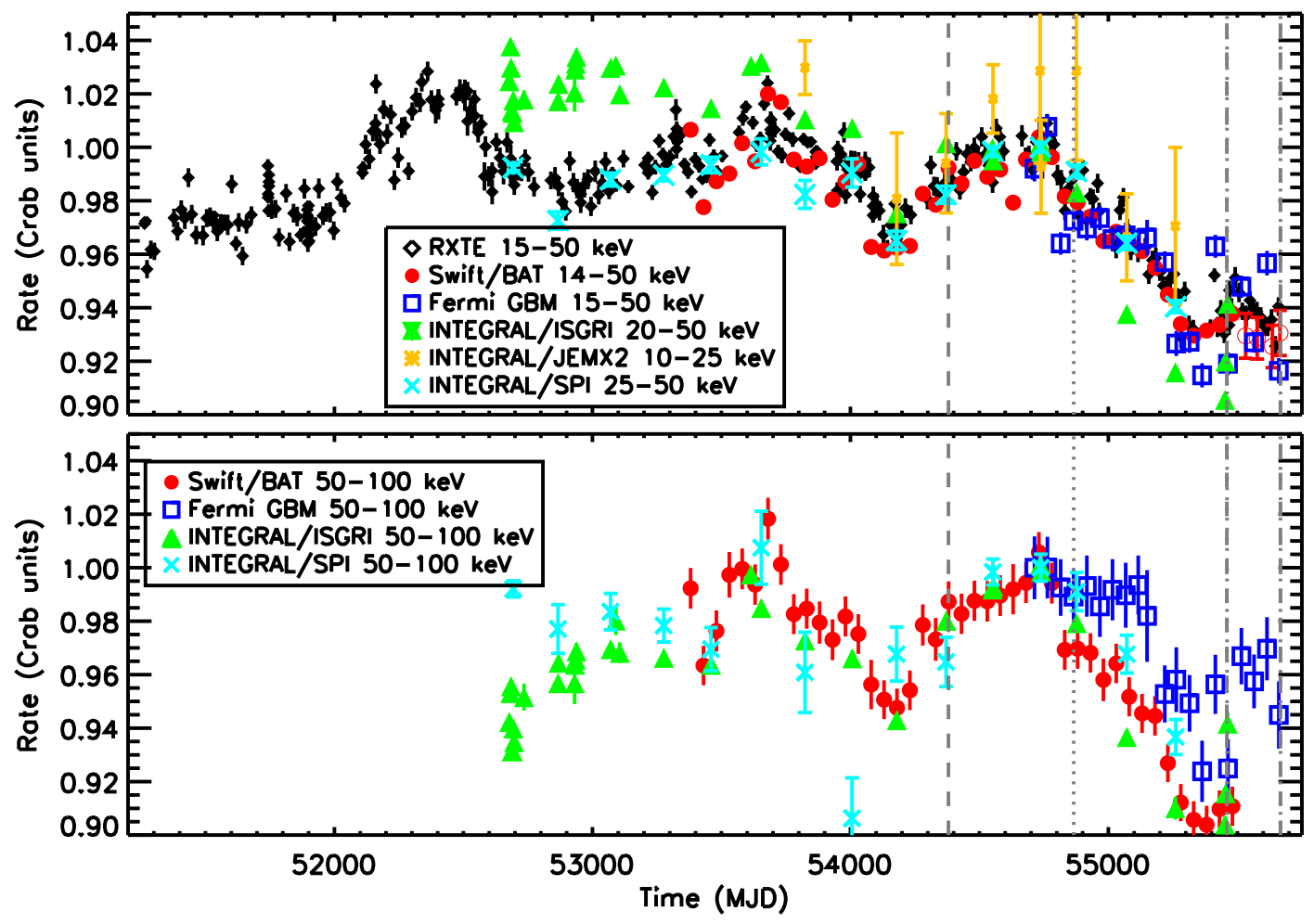

Figure 1: Composite Crab light curves for RXTE/PCA (15-50 keV - black diamonds), Swift/BAT (Top: 14-50 keV, Bottom: 50-100 keV - red filled circles), Fermi/GBM (Top: $15-50 \mathrm{keV}$, Bottom: $50-100 \mathrm{keV}$ - open blue squares), INTEGRAL/SPI (Top: $25-50 \mathrm{keV}$, Bottom: 50-100 keV - light blue crosses), INTEGRAL/ISGRI (Top: 20-50 keV, Bottom: $50-100 \mathrm{keV}$ - green triangles), and INTEGRAL/JEM-X2 (10-25 $\mathrm{keV}$, orange asterisks). Each data set has been normalized to its mean rate in the time interval MJD 5469054790. All error bars (except Swift/BAT) include only statistical errors. Vertical lines mark GeV flares from the Crab observed with AGILE ([7], dashed lines) and Fermi LAT ([8, 9], dotted lines).

onset of rapid spin-up beginning 2010 June 25. The spin period changed by 3 seconds during the sharp rise of the flare. This episode of spin-up resembles two episodes observed with BATSE [12] and one observed with GBM in 2009 January.

\section{Discussion}

In this paper, we have described highlights of serendipitous science from the Fermi GBM, specifically from the Earth occultation monitoring and accretion powered pulsar monitoring projects. This science is beyond the primary gamma ray burst science of GBM and is supported by the Fermi Guest Investigator program. It is made possible by GBM's large field-of-view (the entire unocculted sky), GBM's broad energy range (8-1000 keV for the NaI detectors), and GBM's time resolution (0.256-s for accreting pulsar monitoring.) GBM's near continuous data with good time resolution enables monitoring of accretion-powered pulsar spin-frequencies, source state changes [13], quasi-periodic oscillations [10], and detections of X-ray bursts [15]. All-sky monitors are valuable not only as triggers for the main instrument, but also for stand alone science. 


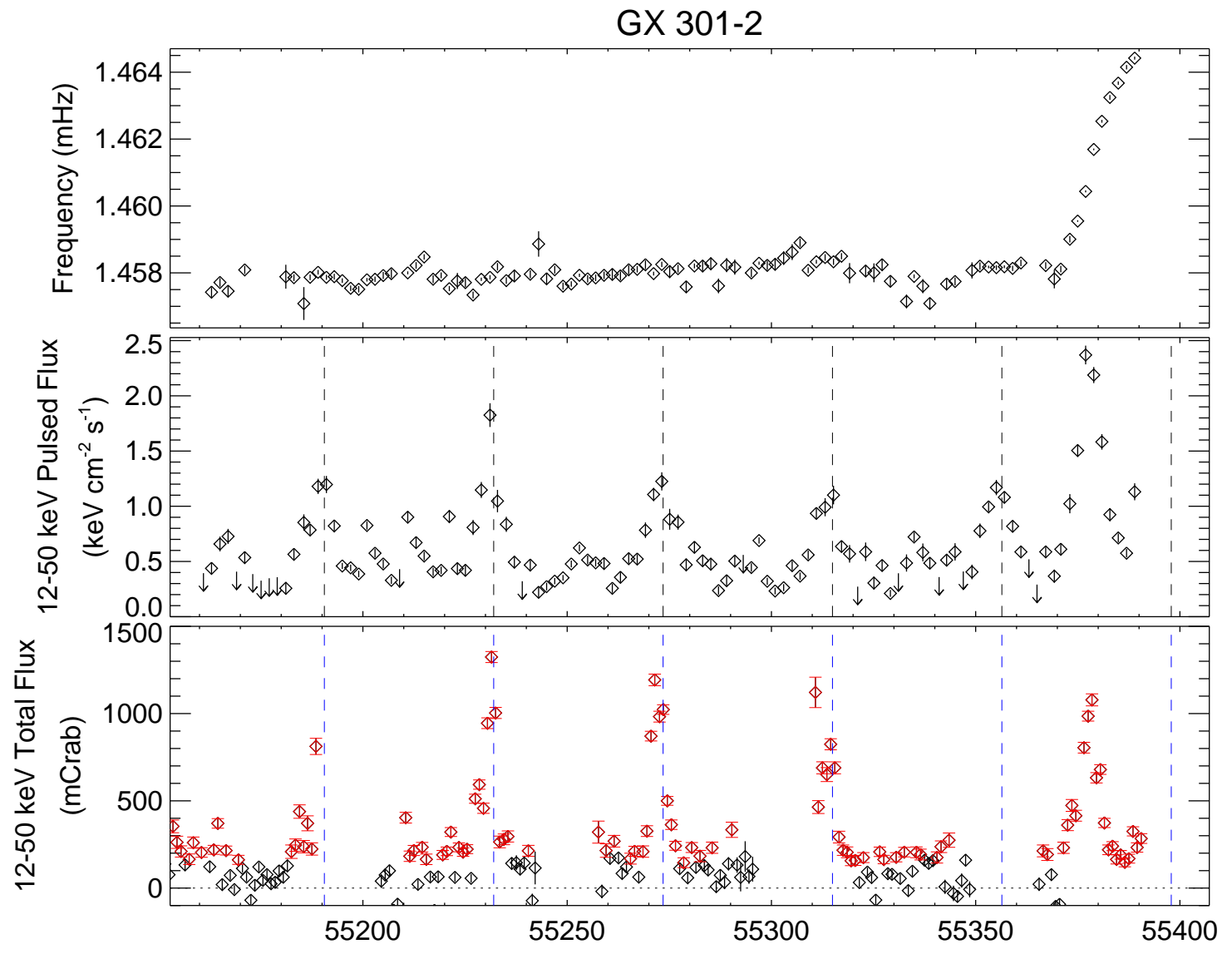

Figure 2: Top: GX 301-2 spin frequency. Center: GX 301-2 pulsed flux in the 12-50 keV band. Bottom: GX 301-2 total flux in the 12-50 keV band measured with GBM Earth occultation. Downward arrows in the center panel are upper limts. Red points in the bottom panel indicate 3- $\sigma$ or better statistical significance. Gaps in the bottom panel are because GX 301-2 is a high declination source and is not always Earth occulted, meaning that GX 301-2 is continuously visible to GBM for a portion of Fermi's precession cycle, increasing the exposure time for pulsar monitoring observations..

\section{References}

[1] Atwood, W.B. et al. 2009, ApJ,697, 1071

[2] Meegan, C.A. et al. 2009, ApJ, 702, 791

[3] Wilson-Hodge, C.A. et al. 2009, Proc. Fermi Symposium, eConf C091122, [astro-ph/0912.3831]

[4] Case, G.L. et al. 2011, ApJ, 729, 105

[5] Wilson-Hodge, C.A. et al. 2011, ApJ, 727, L40

[6] Roques, J.P. et al. 2003, $A \& A, 411,91$

[7] Tavani, M. et al. 2011, Science, 331, 736

[8] Abdo, A.A. et al. 2011, Science, 331, 739

[9] Buehler, R. et al. 2011, ATel \# 3276 
[10] Finger, M.H. et al. 2009, Proc. Fermi Symposium, eConf C091122, [astro-ph/0912 . 384 7]

[11] Finger, M.H. et al. 2010, ATel \# 2712

[12] Koh, D.T. et al. 1997, ApJ, 479, 933

[13] Wilson-Hodge, C.A. \& Case, G. 2010, ATel \#2721

[14] Finger, M.H., Wilson-Hodge, C.A., Camero-Arranz, A. 2009, ATel \#2346

[15] Linares, M. 2011, in Proceedings of The First Year of MAXI: Monitoring Variable X-ray Sources 